\title{
J122015
}

\section{Analysis and Design Optimization of Heavy Goods Vehicle to Minimize Injury}

\section{Risks to Pedestrians}

\author{
Faiz Redza Ramli ${ }^{1 *}$, Koetsu Yamazaki ${ }^{2^{*}}$ \\ ${ }^{1}$ Graduate School of Natural Science and Technology, Kanazawa University, 920-1192, Kanazawa, Japan \\ * Corresponding author: faredza@stu.kanazawa-u.ac.jp \\ ${ }^{2}$ Kanazawa University, 920-1192, Kanazawa, Japan
}

\begin{abstract}
The pedestrian protection against vehicle collision is one of a highly crucial issue in the vehicle safety design. However, only a small number of studies have been conducted in the area of pedestrian collision involving the Heavy Goods Vehicle (HGV) because it usually have aggressive frontal design and high mass that might not be safe to the pedestrian bodies during collision. This paper addresses the optimizations of HGV front structure by two design concepts to reduce the pedestrian injuries. The first concept is by an additional component under a bumper called lower bumper stiffener and the next concept is an attached airbag in front of the HGV panel. The HGV-to-pedestrian collision was simulated by using the crash analysis solver MADYMO in which the pedestrian models of child facing to the left of HGV was expected to survive the HGV impact speed of less than $25 \mathrm{~km} / \mathrm{h}$. The design parameters of bumper and airbag were varied by the adoption of the RSM techniques with the Latin Hypercube Sampling (LHS) and the pedestrian injuries of head injury criterion $(H I C)$, thorax cumulative acceleration $\left(C_{3 \mathrm{~ms}}\right)$, and peak femur loads for both legs were taken as the objective functions. Based on the result, both of the concepts were able to reduce the child injuries and the airbag concept had shown better results especially in minimizing the head injuries.
\end{abstract}

Keywords: Heavy Goods Vehicle(HGV), Pedestrian Safety, Collision Damage Estimation, Multi-objective Optimization, MADYMO

\section{Introduction}

Based on the study conducted by World Health Organization (WHO) [1] an estimation of 1.2 million people died from road traffic accident in 2008. Estimation of the global traffic fatalities in [2] found that pedestrian fatalities are high especially in the low and the middle income countries. In the United States of America alone, the children under the age of 16 accounted for about 10 percent of the pedestrian fatalities [3]. Although less than $5 \%$ of pedestrian fatalities involved in the heavy trucks accidents compare to the accidents of other vehicles [4], the importance of producing a safer design of heavy vehicle should not have been ignored.

The Heavy Goods Vehicle (HGV) which can be defined as a truck or a lorry that has gross combination mass of over 3,500 kgw is not heavily approach in pedestrian safety. This is because of its problematic nature of having aggressive front geometry and a higher risk of pedestrian run over. So far only few passive system improvements of HGV were studied to reduce pedestrian fatality and serious injury. Chawla et al. [5], Feist et al. [6] and Hamacher et al. [7] had suggested various change to the front of truck such as altering bumper height, bumper offset and the use of EPP foam as an energy absorption front. In pedestrian safety involving automobiles, lower stiffener was introduced to reduce the bending angle of leg and the tibia acceleration so that the pedestrian kinematics after collision is improved. In addition, a pedestrian airbag for automobiles to protect pedestrian head injury has also been invented in automotive safety. In this system, the car's hood will be push upward and an airbag under the base of the windshield will be deployed to protect head injuries. The system uses high sensitivity radar and infrared technology to pre-detect a collision and inflates quickly enough to cushion the impact.

The aim of this research is to reduce the child injuries when the child pedestrian is collided against the HGV in the MADYMO multi-body environments. Two suggested design concepts of HGV airbag in front of front panel, respectively, were adopted. The first concept is by an additional component under a bumper called lower bumper stiffener and the next concept is an attached airbag in front of the HGV panel. The child lateral impact facing the left front surface of HGV was chosen as the child case to be studied. The scope of this

${ }^{* 1}$ 准員, 金沢大学 自然科学研究科 人間 - 機械科学専攻

*2 正員, 金沢大学理工学研究域 (广920-1192 金沢市角間町)

E-mail: faredza@stu.kanazawa-u.ac.jp

[No.12-1] 日本機械学会 2012 年度年次大会講演論文集 [2012.9.9-12, 金沢] 
study is only focused on the duration of impact between child pedestrian model with the HGV model, respectively. Therefore, the results of this study will ignore all the post impact cases such as impact of child with road or vehicle run-over onto the child after collision.

\section{Methodologies}

\subsection{Child pedestrian model}

The child pedestrian used in this study were realized by a 6 year old pedestrian child model developed by MADYMO. This MADYMO pedestrian model is an ellipsoid human model developed by MADYMO and the model was built based on the data of Westem European population. The height and weight of the child pedestrian models are $1.17 \mathrm{~m}$ and $0.23 \mathrm{kgw}$, respectively. Each child pedestrian model is developed by 52 rigid bodies and is described by 64 ellipsoids to replicate the human bodies. The ellipsoids of the model act as several individual body parts such as head, torso or upper leg where the stiffness, shape, size, mass and inertia are predefined based on the study by MADYMO. In addition, the pedestrian model can measure the injured loads acted on the bodies, if any external impact acted on it. The MADYMO pedestrian models have been validated which can be referred in the MADYMO Human Models Manual [8]. A study by Anderson et al. [9] demonstrated that the walking posture of the pedestrian before collision with vehicle front would influences the pedestrian kinematics and the pedestrian injuries after the impact. Thus, the child pedestrian's initial $80 \%$ period of gait cycle from Untariou [10] which is facing left such as shown in Fig.1, was chosen as the child case to be studied

Standard injury threshold parameters have been recommended by recognized bodies such as EuroNCAP to the limitation of pedestrian body sustained before undergoing mechanical and physiological changes. For head injury, the HIC, which is a measure of the possibility of head injury occurs from an impact, is utilized. The HIC equation is stated as follows;

$$
H I C=\left\{\left[\frac{1}{t_{2}-t_{1}} \int_{t_{1}}^{t_{2}} a d t\right]^{2.5}\left(t_{2}-t_{1}\right)\right\}_{\max }
$$

where $t_{1}$ and $t_{2}$ are the initial and final times (in seconds) of the interval duration when the $H I C$ attains a maximum value, and acceleration of head is measured in $\mathrm{g}$. The maximum time duration of HIC $t_{2}-t_{1}$, is usually limited to $15 \mathrm{~ms}$ and $H I C$ values greater than 1,000 have been considered unacceptable. The thorax deceleration was used as indicator for the thorax injury by a common criterion, the thorax Cumulative $3 \mathrm{~ms}$ Acceleration $\left(C_{3 \mathrm{~ms}}\right) . C_{3 \mathrm{~ms}}$ can be defined as the highest acceleration level that can be sustained for at least $3 \mathrm{~ms}$ or longer by the center of gravity of thorax. A tolerance level of $60 \mathrm{~g}$ was used to indicate a severe chest injury. Lastly, for the upper leg injuries, a peak force value acted on femur must be less than $10 \mathrm{kN}[11]$

\subsection{HGV model}

The baseline HGV model was developed by three rigid bodies of ellipsoids of front panel, bumper foam and lower stiffener shown in Fig. 2. The front panel ellipsoid was assumed to perform as the HGV front components such as grille and hood. The HGV's windshield was ignored in this study since it was assumed that the height of the windshield was high enough that no contact occurs with child pedestrian during impact. In addition, the force-displacement curve for the HGV front structure of Ref.(5) was implemented in the study. Since the lower stiffener had not been used in the HGV before, a car bumper and lower stiffener from Ref.(12) are used in this study. The force-displacement characteristics used for the ellipsoids were kept constant throughout the study. The initial location of the lower stiffener is arranged based on the height of child model. For this reason, the initial location of the bumper foam and the location of lower stiffener were set at $500 \mathrm{~mm}$ and $200 \mathrm{~mm}$ above the ground, respectively. In addition, both of the bumper foam and lower stiffener were positioned $100 \mathrm{~mm}$ in front of the HGV front panel.

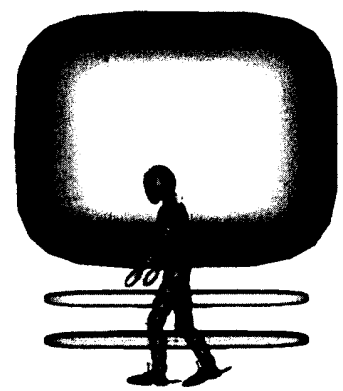

Figure 1 Lateral impact of HGV-child pedestrian facing left

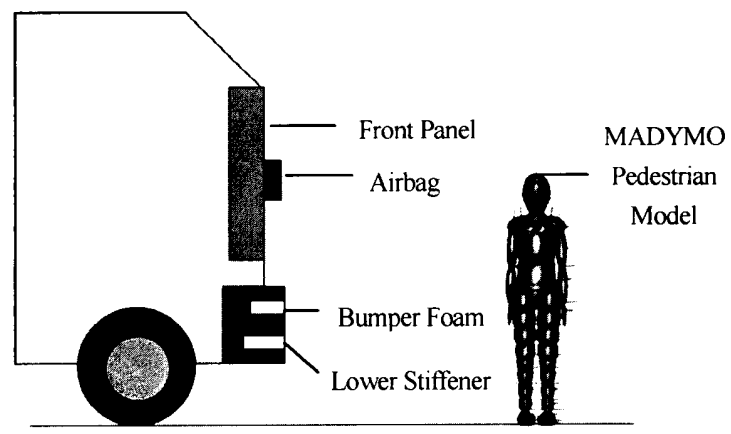

Figure 2 HGV-pedestrian model setup 


\subsection{Design Optimization of HGV Front}

Two design concept of HGV front structure were considered in this paper. The Design Concept 1 shown in Fig.3(a) was intended to investigate how the variation parameters of bumper and lower stiffener could reduce the child pedestrian injuries. While the Design Concept 2 considered an airbag attached to the front of HGV panel such as shown in Fig.3(b). For the Design Concept 1, the lower stiffener protrusion $x_{\mathrm{s}}$ and bumper protrusion $x_{\mathrm{b}}$ were varied their positions forward and back between $-100 \mathrm{~mm}$ and $100 \mathrm{~mm}$. While the lower stiffener height $y_{\mathrm{s}}$ and bumper height $y_{\mathrm{b}}$ were moved downwards and upwards from the initial position $-100 \mathrm{~mm}$ and $100 \mathrm{~mm}$. While for the Design Concept 2, the same HGV baseline model but with an added airbag was positioned in the front and mid-center line of HGV front panel. The MADYMO finite elements airbag model [10] that was used to protect car passenger, was implemented in this setup. The location of airbag in z-direction was adjusted based on the height of the child model. The maximum height of airbag is based on the height of child where the distance of $1.0 \mathrm{~m}$ from the ground was chosen. The parameters were varied by $\pm 200 \mathrm{~mm}$ in order to observe the effect of airbag impact with pedestrian upper injuries. Other airbag parameters were maintained from the basic MADYMO model. For the bumper, the bumper protrusion $x_{\mathrm{b}}$ were varied their positions forward and back between $-100 \mathrm{~mm}$ and $100 \mathrm{~mm}$. and the bumper height $y_{\mathrm{b}}$ were moved downwards and upwards from the initial position $-100 \mathrm{~mm}$ and $100 \mathrm{~mm}$.

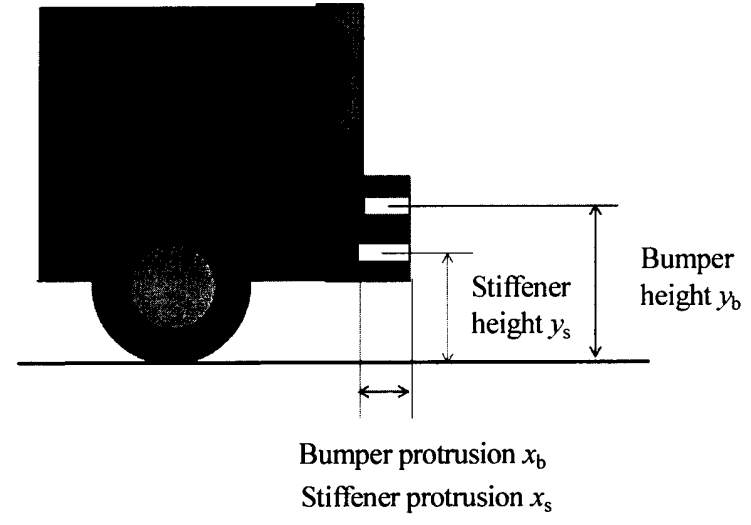

(a) Design Concept 1

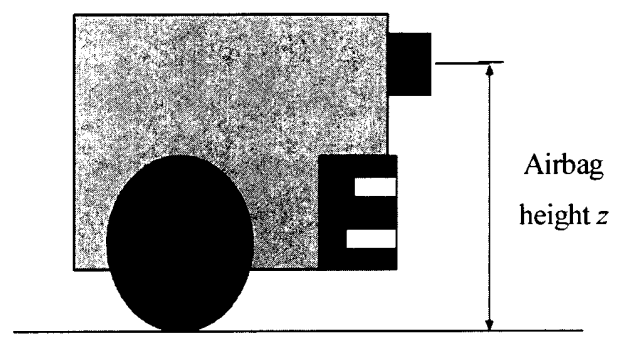

(b) Design Concept 2

Figure 3 HGV bumper variables Design Concept 1 and airbag parameters Design Concept 2

\subsection{Optimum Design Problem Definition}

For safety consideration, the head injury criterion $H I C, C_{3 \mathrm{~ms}}, F_{\mathrm{L}}$ and $F_{\mathrm{R}}$ loads should be less than the values decided in the safety regulation. A general optimization problem for both of design cases stated in the previous sub-section can be express as follows;

Minimize injury

$$
\mathrm{F}(\mathrm{x})=\left[H I C, C_{3 \mathrm{~ms}}, F_{\mathrm{L}}, F_{\mathrm{R}}\right]
$$

Subjected to

For Design Concept 1

$$
\begin{aligned}
& 0(\mathrm{~mm}) \leq x_{\mathrm{s}} \leq 200(\mathrm{~mm}) \\
& 0(\mathrm{~mm}) \leq x_{\mathrm{b}} \leq 200(\mathrm{~mm}) \\
& 100(\mathrm{~mm}) \leq y_{\mathrm{s}} \leq 300(\mathrm{~mm}) \\
& 400(\mathrm{~mm}) \leq y_{\mathrm{b}} \leq 600(\mathrm{~mm}) \\
& 0(\mathrm{~mm}) \leq x_{\mathrm{b}} \leq 200(\mathrm{~mm}) \\
& 400(\mathrm{~mm}) \leq y_{\mathrm{b}} \leq 600(\mathrm{~mm}) \\
& 800(\mathrm{~mm}) \leq z \leq 1200(\mathrm{~mm})
\end{aligned}
$$

For Design Concept 2

\subsection{Solution method}

The design problem including the objectives, constraints and design variables were defined such as in Eqs. (2-9). Twenty sampling points was then selected based on the LHS in conducting the computer simulations of HGV-Pedestrian collision. The responses for the pedestrian injuries of $H I C, C_{3 \mathrm{~ms}}, F_{\mathrm{L}}$ and $F_{\mathrm{R}}$ obtained were used to construct quadratic regression models to approximate the solutions. Afterwards, the fitting accuracy of the models is validated by statistical analysis techniques. The satisfied quadratic models for each $H I C$, $C_{3 \mathrm{~ms}}, F_{\mathrm{L}}$ and $F_{\mathrm{R}}$ were then solved by multi-objective optimization technique. Because of the collision involved the whole pedestrian 
bodies, the weight of the significance injuries for each bodies is considered. Desirability functions [13] for each models were made separately with the significance weights and the threshold injuries assigned to them. Then the geometric mean of the individual desirability provides the solution to the optimal parameters to minimize the pedestrian injuries.

\section{Result and Discussion}

\subsection{Optimization Results of Design Concept 1}

Table 1 showed the difference values of $H I C, C_{3 \mathrm{~ms}}, F_{\mathrm{L}}$ and $F_{R}$ injuries between baseline model and the Design Concept 1. From these results, by moving the bumper forward by $126 \mathrm{~mm}$ and positioning the bumper at $516 \mathrm{~mm}$ above the ground, the optimal protection to the head, the thorax and the leg injuries were attained. In addition, optimum values of injuries were also obtained when changing the position of lower stiffener nearer to the front panel and downward to the ground. The most improved condition is the thorax injury, where the value of $C_{3 \mathrm{~ms}}$ had decreased significantly from higher than tolerance of $69 \mathrm{~g}$ to the value of $49 \mathrm{~g}$.

Fig.4 shows the illustration of impact between HGV and the child pedestrian. In Fig.4(a), when the child was hit by the HGV which the bumper protrusion and bumper stiffener is $100 \mathrm{~mm}$ from the front HGV, almost all child bodies is hit directly by the front of HGV. Whereas, in Fig..4(b), the HGV which the bumper protrusion and bumper stiffener is $200 \mathrm{~mm}$ from the front HGV, the chest is not hit directly because there is an increased distance between the bumper and the HGV front panel. With the additional space between the bumper and the front of HGV, the child body has enough time to shift its position and avoids a direct hit between the HGV front panel and the child bodies. Thus the $C_{3 \mathrm{~ms}}$ values had decreased. Also from Fig.4, it is undesirable to increasingly shift the bumper more than $200 \mathrm{~mm}$. Since the HGV in this study consists of only three ellipsoids the gap between the bumper foam and front panel is too large. So no contact between the upper bodies and the front panel will be existed. Hence, the maximum value of $200 \mathrm{~mm}$ for the bumper protrusion is considered suitable for this study.

Furthermore, both of the leg injuries were fully dependent on the position of bumper height since the measurements of upper legs were used in this study. Because of this reason, the optimal values of $F_{\mathrm{L}}$ and $F_{\mathrm{R}}$ were different from the values compared to the baseline model. However, the values are low as compared to the threshold values of $10 \mathrm{kN}$.

Table 1 Results of optimal design

\begin{tabular}{c|c|c|c|c|c}
\hline Concepts & Optimal design variables & $H I C$ & $C_{3 \mathrm{~ms}}(\mathrm{~g})$ & $F_{\mathrm{L}}(\mathrm{kN})$ & $F_{\mathrm{R}}(\mathrm{kN})$ \\
\hline Baseline model & & 416.9 & 69.0 & 1.25 & 0.7 \\
\hline $\begin{array}{c}\text { Lower stiffener concept } \\
\text { (Design Concept 1) }\end{array}$ & $\begin{array}{l}x_{\mathrm{s}}=0 \mathrm{~mm}, \quad x_{\mathrm{b}}=126 \mathrm{~mm}, \\
y_{\mathrm{s}}=100 \mathrm{~mm}, \quad y_{\mathrm{b}}=516 \mathrm{~mm}\end{array}$ & 404.2 & 49.6 & 0.7 & 0.9 \\
\hline
\end{tabular}

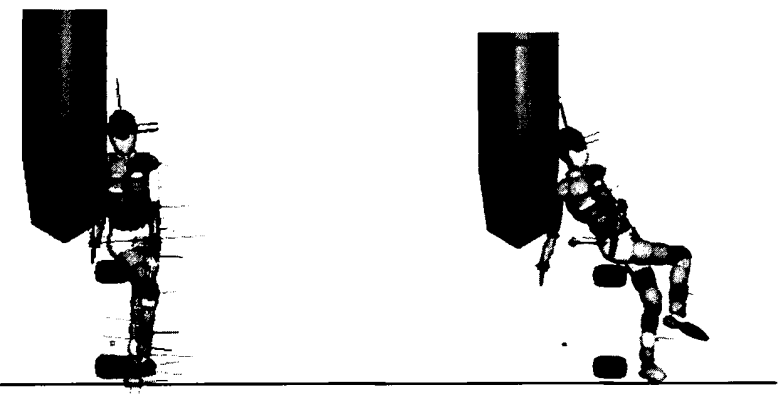

(a) Bumper protrusion, $x_{\mathrm{b}} 100 \mathrm{~mm}$

(b) Bumper protrusion, $x_{\mathrm{b}} 200 \mathrm{~mm}$

Figure $4 \mathrm{HGV}$ impact between child when bumper protrusion is varied

\subsection{Optimization Results of Design Concept 2}

For the analysis of the airbag concept, Table 2 showed the difference values between baseline model and the Design Concept 2. The airbag height $z$ obtained is $0.97 \mathrm{~m}$. Since the height of the child pedestrian is $1.17 \mathrm{~m}$, the location of the airbag is considered sensible as the airbag is good enough to protect both of the head and thorax injuries. Whereas, the location of the bumper installed is $520 \mathrm{~mm}$ from the ground and the bumper protrusion obtained is $173 \mathrm{~mm}$. The location of bumper protrusion has influenced the result of the $H I C$, since the airbag sensor will detect the crash signal when the bumper hit the leg. If the bumper is too near to the front panel, the airbag might not be able to be exploded after the detection. For this reason, it is considered that the optimum value of $173 \mathrm{~mm}$ protrusion gives a good result to 
the protection of upper injuries. From the results of the injuries, the values of $H I C$ and $C_{3 \mathrm{~ms}}$ have been improved. However, minor changes were observed from the results of $F_{\mathrm{L}}$ and $F_{\mathrm{R}}$, because these leg injuries are mostly influenced by the impact of bumper and lower bumper stiffener, and not by the airbag. All of the results are below the threshold values, which mean that the airbag with lower stiffener bumper is considered able to protect the pedestrian effectively.

Table 2 Results of Optimal Design

\begin{tabular}{c|c|c|c|c|c}
\hline Concepts & Optimal design variables & $H I C$ & $C_{3 \mathrm{~ms}}(\mathrm{~g})$ & $F_{\mathrm{L}}(\mathrm{kN})$ & $F_{\mathrm{R}}(\mathrm{kN})$ \\
\hline Baseline model & & 416.9 & 69.0 & 1.25 & 0.7 \\
\hline $\begin{array}{c}\text { Airbag concept } \\
\text { (Design Concept 2) }\end{array}$ & $\begin{array}{l}x_{\mathrm{b}}=173 \mathrm{~mm}, \quad y_{\mathrm{b}}=520 \mathrm{~mm} \\
z=970 \mathrm{~mm}\end{array}$ & 121.3 & 39.6 & 1.1 & 0.7 \\
\hline
\end{tabular}

\section{Conclusions}

In this paper, two concepts of bumper with lower bumper stiffener and airbag, which had been studied in the pedestrian safety of automobiles, had been successfully applied and optimized for the $25 \mathrm{~km} / \mathrm{h} \mathrm{HGV}$ to child pedestrian collision. For lower bumper stiffener concepts, the child pedestrian injuries were minimized by positioning the front geometries of bumper and lower bumper stiffener. Whereas, by locating the exact airbag position and the parameters of airbag had improved the pedestrian injuries. Comparing the two concepts, the airbag had shown better results in reducing pedestrian injuries, especially the head injuries. The information provided in this paper might be used to aid the understanding towards designing a better HGV front in the future by considering the child pedestrian safety aspects.

\section{References}

1. World Health Organization The ten leading causes of death by broad income group Fact sheet 310, 2008. Available from URL:http://www.who.int/mediacentre/factsheets/fs310/en/index.htm

2. Naci, H., Chisholm, D. and Baker, T. D. Distribution of Road Traffic Deaths by Road User Group: a Global Comparison. Journal of Traffic Injury Prevention, 2009, Vol.15, pp.55-9.

3. NHTSA (2008) National Pedestrian Crash Report Available From URL: http://wwwnrd.nhtsa.dot.gov/Pubs/810968.pdf

4. Paulozzi, LJ, United States pedestrian fatality rates by vehicle type Journal of Injury Prevention 2005 August; 11(4): 232-236

5. Chawla, A., Sharma, V., Mohan, D. and Kajzer, J., Safer truck front designs for pedestrian impacts, Proceedings 1998 International IRCOBI Conference on the Biomechanics of Impact 1998.

6. Feist F., Guglera J., Giordab A., Avalleb M. and Puppini R., Improvements to the protection of vulnerable road users: Retrofittable, energy-absorbing front end for heavy goods vehicles, Int Journal of Crashworthiness, 2008 13(6), 609-627.

7. Hamacher M., Fassbender S., Fest F. and Gugler J., Modification of a truck front for improved kinematics in run over, Proceedings of the 21st International Technical Conference of Enhanced Safety Vehicles Conference (ESV), International Congress Center Stuttgart, Germany, 2009.

8. MADYMO Theory Manual Release 7.0, TNO, 2008.

9. Anderson R.W.G, Long A.D., Serre T. and Masson C., Determination of boundary conditions for pedestrian collision reconstruction. Proceedings of the ICrash Conference, Kyoto, Japan. 2008.

10. Untaroiu, C.D., Meissner, M., Crandall, J.R., Takahashi, Y., Okamoto, M. and Ito, O. Crash Reconstruction of Pedestrian Accidents using Optimization Techniques. International Journal of Impact Engineering 2009 36(2): 210-219.

11. European New Car Assessment Protocol (EuroNCAP) Pedestrian Testing Protocol version 4.1, Available Online, http://www.ancap.com.au/admin/uploadfiles/EuroNCAP_Assessment_Protocol_V4.1.pdf.

12. Untaroiu C.D., Shin J., Crandall J., Fredriksson R, Bostrom O, Takahashi Y., Akiyama A., Okamoto M. and Kikuchi Y. Development and Validation of Pedestrian Sedan Bucks using Finite Element Simulations; Application in Study the Influence of Automatic Braking on the Kinematics of Pedestrian involved in Vehicle Collision, International Journal of Crashworthiness 2010 15(5), 491-503.

13. Derringer, G and Suich, R. Simultaneous Optimization of several response variables. Journal of Quality Technology 1980 12, $214-219$. 